\title{
Internal models for bi-manual tasks
}

\author{
Alice G. Witney * \\ Department of Zoology, University of Cambridge, Downing Street, Cambridge CB2 3EJ, UK
}

\begin{abstract}
Co-ordinated bi-manual actions form the basis for many everyday motor skills. In this review, the internal model approach to the problem of bi-manual co-ordination is presented. Bi-manual coordinative tasks are often regarded as a hallmark of complex action. They are often associated with object manipulation, whether the holding of a single object between the two hands or holding an object in each hand. However, the task of movement and control is deceptively difficult even when we execute an action with a single hand without holding an object. The simplest voluntary action requires the problems of co-ordination, timing and interaction between neural, muscular and skeletal structures to be overcome. When we are making a movement whilst holding an object, a further requirement is that an internal model is able to predict the dynamics of the object that is being held as well as the dynamics of the motor system. There has been extensive work examining the formation of internal models when acting in novel environments. The majority of studies examine uni-lateral learning of a task generally to the participant's dominant hand. However, many everyday motor tasks are bi-manual, and the existing findings regarding the learning of internal models in uni-manual tasks and their subsequent generalization highlights the complexities that must underlie the formation of bi-manual tasks. Our ability to perform bi-manual tasks raises interesting questions about how internal models are specified for co-ordinative actions, and also for how the motor system learns to represent the properties of objects.

(c) 2004 Elsevier B.V. All rights reserved.
\end{abstract}

\footnotetext{
* Tel.: +44 1223 335561; fax: +44 1223336676 .

E-mail address: agw29@cam.ac.uk

0167-9457/\$ - see front matter (C) 2004 Elsevier B.V. All rights reserved. 


\section{Introduction}

The co-ordination of bi-manual actions is an important problem to understand as it provides a model for how complex motor skills are learned and executed. We show tremendous modularity in the range of bi-manual movements that we can perform, and this extends to the co-ordination of actions with hand-held objects. A single object can be held and manipulated between the hands, but we can quickly switch to holding two different objects in each hand. Dynamic systems approaches are particularly dominant theoretically in explaining the problem of bi-manual co-ordination (Kelso, Southard, \& Goodman, 1979). A second approach to this problem has been information processing (Swinnen, Walter, Lee, \& Serrien, 1993; Swinnen \& Wenderoth, 2004). Here the internal model perspective to bi-manual co-ordination is reviewed.

The concept of internal models of the motor system, that is, representations within the central nervous system that simulate the naturally occurring transformations between sensory signals and motor commands, has become a central theoretical framework to our understanding of the neural control of movement (Wolpert \& Ghahramani, 2000; Wolpert, Ghahramani, \& Flanagan, 2001). Most studies of internal model learning have examined learning in the dominant hand (Goodbody \& Wolpert, 1998; Shadmehr \& Brushers-Krug, 1997; Shadmehr \& Mussa-Ivaldi, 1994). However, motor learning in the dominant hand is thought to differ from that in the non-dominant hand due to the asymmetry in our hand preferences, and underlying neural control (Sainburg \& Kalakanis, 2000). Recently studies have examined the generalization of internal model learning between the dominant and non-dominant side, and found a variety of differences dependent on the experimental task (Sainburg \& Wang, 2002; Wang \& Sainburg, 2004). The predictive control of grasp forces provides a useful method of examining the learning of internal models for bimanual actions and how internal models for co-ordinative actions develop. The particular focus of this paper is the requirements for the learning of internal models for bi-manual actions, and the problems that may be inherent in their construction.

\section{The problem of learning an internal model for bi-manual object manipulation: Constituent elements}

(A) Handedness. We have a natural asymmetry in our movement control. This asymmetry may influence many everyday bi-manual tasks. Each hand commonly has a favoured role in the task, for instance as the supportive or acting hand.

(B) Learning with the dominant and non-dominant hand:

(i) There is an asymmetry in generalization of learning between dominant and non-dominant hands. Studies examining the generalization of learning in novel kinematic and dynamic environments show that although the pattern of generalization varies dependent on task, asymmetries are generally present. 
(ii) Does the generalization of learning bi-manual tasks occur in an Intrinsic or Extrinsic co-ordinate system? For an object representation to be formed, generalization should be in an extrinsic coordinate system.

(iii) To what extent is the movement control of the two hands independent?

(C) How does a representation of a single object being held bi-manually emerge? A number of factors may be considered to be important in the formation of an object representation, and the relative importance of these factors should be considered. For instance, how important is an efference copy compared with synchronicity of loading and knowledge of the object properties?

\section{Internal models of the motor system}

Computationally, the motor system can be regarded as a dynamical system, whose inputs are the motor commands from the 'controller' within the Central Nervous System (CNS) and outputs are sensory feedback. Knowledge of just the input to a dynamical system is not enough to predict the output from the system. However, when the 'state' of the system is known in addition to the motor command, the behaviour of the system can be predicted. The state therefore contains all the relevant time-varying information needed to predict or control the future of the system. For example, to predict the effects of applying a torque around the knee joint, it is also necessary to know the configuration and motion of other body segments. One of the problems the motor system has to overcome is how to estimate the state from the motor command and sensory feedback.

Representations of the sensori-motor transformations between motor and sensory variables are thought to occur within the CNS. Such internal models are systems that simulate the behaviour of natural processes and transform sensory signals to motor commands and motor commands to sensory signals. There are two main groups of internal models, known as forward and inverse models (Jordan, 1996; Wolpert, 1997; Wolpert \& Ghahramani, 2000; Wolpert et al., 2001).

The forward model is a causal representation of the motor apparatus that represents the behaviour of the motor system in response to outgoing motor commands (Kawato, Furawaka, \& Suzuki, 1987; Wolpert, 1997; Wolpert \& Flanagan, 2001). That is, forward models capture the causal relationship between actions and outcome, as signaled by an efference copy of the motor command (Sperry, 1950; Von Holst, 1954). Inverse models simulate the transformation from outputs to inputs, so that the intended outcome of the movement is the basis for the generation of the appropriate motor commands. Unlike the forward model, the inverse model is not necessarily unique, there are often many possible ways that a movement outcome can be obtained, and the transformation can be one-to-many.

For internal models to be functional, it is essential that they are not static, and can be modified as new environments are encountered, for example novel objects are 
manipulated, and also to take into account changes that occur in the properties of the arm due to growth and injury. The ability to learn and use new internal models is, therefore, a fundamental property of the motor system (Shadmehr \& BrushersKrug, 1997; Wolpert, 1997; Wolpert \& Ghahramani, 2000).

Recent computational frameworks extend these ideas by proposing a multi-layered controller (Wolpert, Doya, \& Kawato, 2003). The lowest level of this model has paired forward and inverse models that are action specific, and therefore estimate and control the movement for a specific object using a specified effector, for instance the grasp and lift of an object with the right hand (Haruno, Wolpert, \& Kawato, 2001; Wolpert \& Kawato, 1998). Moving up the hierarchy of the multi-layered controller, each level receives information from the level below it with the representation at each level becoming increasingly abstract. Therefore, the representation will become increasingly independent both of specific trajectories and effectors. The highest levels of this hierarchical controller will therefore represent behavioural goals rather than a detailed specification of how they are implemented. Such a framework may become particularly important for our understanding of how bi-manual internal models are learned, in particular how we develop a representation for a single object being manipulated between two hands, or the recognition that the same environment is being acted in to the left and to the right.

\subsection{Handedness}

A number of recent studies have focussed on the differences between the formation of internal models in relation to handedness. Handedness is the manual asymmetry whereby one hand is used in favour of the other hand in the performance of skilled uni-manual tasks (Sainburg \& Kalakanis, 2000). Combined with studies on brain-damaged patients and functional imaging this provides an insight into possible underlying asymmetries in neural control (Haaland \& Harrington, 1996). Handedness is frequently defined through the application of a multiple part Edinburgh Inventory, where the hand preferred for use in a number of everyday tasks is elicited (Oldfield, 1971). Examination of this Inventory suggests that the definition of handedness can in fact be extended from the preferred hand in unimanual tasks, as handedness can be defined in many bi-manual tasks. In many everyday bi-manual tasks the non-dominant hand is the postural hand with the dominant hand performing the action. This includes items for handedness included in the Edinburgh Inventory, for instance: "Which hand do you cut a loaf of bread with?"

The dominant hand would be classified as the hand that performs the cutting, however the non-dominant hand would be involved in the task; by holding the bread. Many other similar examples of asymmetric bi-manual tasks are easily thought of, for instance when undoing the lid of a jar, again the dominant hand would be used to perform the precision task, whereas the non-dominant hand would stabilize the jar during the task. This role of the non-dominant hand for postural stabilization is a common one in bi-manual tasks, and an important example is the 
"waiter task" as this has been examined experimentally. The waiter task illustrates how a waiter is able to unload plates and bottles from a tray with one hand while the tray is balanced on the contralateral forearm. In this task the waiter would use the arm of the non-dominant hand to balance the tray whilst using their dominant hand to perform the action of carefully lifting a selected glass of the tray and placing the glass in front of the guest. In this situation the position of the waiter's tray does not move, although unloading the tray should provoke an upward arm movement. Such adjustments of the limb compliance dependent on the environmental context are a feature of our motor system (Lacquaniti, Soechting, \& Terzuolo, 1982). Experimentally, the waiter task has been shown to be an important means of examining the prediction of postural disturbances (Duffose, Hugon, \& Massion, 1985; Hugon, Massion, \& Wiesendanger, 1982; Ioffe, Massion, Gantchev, Duffose, \& Kulikov, 1996; Lum, Reinkensmeyer, Lehman, Li, \& Stark, 1992; Massion, 1992; Paulignan, Duffose, Hugon, \& Massion, 1989).

The requirements in the learning of an internal model for the non-dominant hand may differ from that of the dominant hand due to the innate asymmetry in our hand preferences. These natural asymmetries may lead to preferences for the learning of specific aspects of a bi-manual task for the dominant and non-dominant hands.

\subsection{Learning and generalization in dominant and non-dominant hands}

A number of studies suggest further intricacies that may underlie the learning of a bi-manual task. First, the differing abilities demonstrated in our movement control using the dominant compared with the non-dominant hand. Second, the differences demonstrated in generalization patterns to novel force fields. Force fields that perturb movement have been used to examine learning in the control of novel arm dynamics and alterations in visuo-motor mappings are used to examine the learning of novel kinematics. Different aspects of the experienced field are found to generalize dependent on whether the non-dominant or dominant hand first experiences the field, and also dependent on the nature of the task. These studies will now be briefly reviewed.

Much previous work has compared dominant and non-dominant arm performance during aimed movements (Carson, Chua, Elliott, \& Goodman, 1990; Carson, Goodman, Chau, \& Elliott, 1993). These studies have concentrated on the formation of an inverse model, or the appropriate controller for the action to be successfully executed in a novel environment. During such aimed performance tasks, the dominant arm performs better in terms of reaching accuracy if the movements that are required are high precision. Therefore, it may be expected that faster movements, without the requirement of high precision, may show an advantage for the non-dominant arm, but this is not the case. The advantages of the dominant hand in movements that require greater precision has been ascribed to either a greater advantage for an ongoing error correction mechanism during the movement, or alternatively, that movement planning is more efficient for the dominant 
arm. To further examine differences in movement control between the dominant and non-dominant arm, target information has been altered before movement onset. These studies have shown that there are longer reaction times for the dominant arm compared with the non-dominant arm, suggesting inter-limb differences in movement preparation.

The pattern of generalization of learning from the dominant to the non-dominant hand has been examined in tasks where either a novel dynamic (Criscimagna-Hemminger, Donchin, Gazzaniga, \& Shadmehr, 2003; DiZio \& Lackner, 1995) or novel kinematic environment has been experienced (Sainburg \& Kalakanis, 2000; Sainburg \& Wang, 2002; Wang \& Sainburg, 2003). Distinct differences have been found in the ability of a newly formed internal model to generalize to the contralateral hand dependent on the hand that has initially experienced the field. In general, a benefit was found to first experiencing the novel environment with the dominant hand in the learning of force field by the non-dominant hand. However, beyond this some studies show generalization patterns that indicate distinct co-ordination patterns observed between the two arms, for example Sainburg and Kalakanis (2000). This study found that when participants are presented with a novel force field to each arm, although both limbs achieved similar final position accuracy the non-dominant arm showed less effective control of inter-segmental dynamics compared to the dominant arm. This may suggest that the control of the two limbs is mediated by distinct neural mechanisms.

A further question that is raised, based on these task-dependent differences, is whether the pattern of generalization of learning that occurs is in intrinsic, muscle based co-ordinates or extrinsic co-ordinates. An understanding of this occurs is clearly crucial for an understanding of how an internal model for a co-ordinative bi-manual action is learned.

\subsection{Learning of predictions in bi-manual tasks}

Asymmetries in the learning of internal models demonstrate the complexities that underlie the development of an internal model of a bi-manual task. When we are learning an internal model for a single object, the developed representation of an object needs to be invariant to the effector. The learning of an object representation must develop a certain degree of dynamic invariance; a cube must be perceived to be the same cube whether it is held between the hands or between the feet in an analogous way that our handwriting develops a kinematic invariance regardless of the effector. When we learn a novel object do we first learn with one hand the dynamics of the object before we learn that it is the same object with the contralateral hand? There is evidence that there is specificity in our representations of grip force (Salimi, Hollender, Frazier, \& Gordon, 2000). This makes the challenge of forming an internal model for bi-manual object more daunting. This paper will use the development of forward predictive models of grip force during object manipulation to examine the motor learning of a bi-manual task. First, the development of predictive learning will be summarized before demonstrating the impor- 
tance of grip force modulation as a means of examining the development of a prediction of an object representation.

\subsection{Predictive models in bi-manual unloading}

A forward predictive model is thought to be responsible for our ability to predict the consequences of a motor command, enabling postural stability to be maintained. Studies of anticipatory postural adjustments and predictive grip force modulation are thought, even by critiques of internal models (Ostry \& Feldman, 2003) to provide some of the best evidence for their existence.

As previously mentioned, an important example of prediction in a bi-manual task is the waiter task, where the non-dominant arm stabilizes a tray whilst the dominant hand is used to unload it (Duffose et al., 1985; Hugon et al., 1982; Ioffe et al., 1996; Lum et al., 1992; Massion, 1992; Paulignan et al., 1989).

In these unloading tasks, one arm is held in a horizontal position whilst supporting a $1 \mathrm{~kg}$ weight. This weight is unloaded either by the experimenter's hand (imposed unloading) or by the participant's other hand in response to a tone (voluntary unloading). The EMG activity from the brachioradialis of the postural arm, and from the biceps of the active arm are recorded (Hugon et al., 1982). This has enabled a comparison of the latency of the EMG from the postural arm and from the active arm, and therefore a measure of the anticipatory nature of the response. The degree of displacement of the postural forearm is a further measure that has been used to determine whether a predictive response has occurred. Minimal disturbances reflect that a prediction of unloading was available whilst larger disturbances suggest anticipation was not possible.

The position of the weight-supporting forearm was found to change very little when the weight is taken off by the contralateral hand. EMG recordings have shown that an inhibition in the brachioradialis muscle precedes the onset of the change in force caused by the opposite hand. This indicates that an anticipatory postural adjustment occurred prior to the unloading so that the position of the loaded hand remains unchanged (Duffose et al., 1985; Massion, 1992). When the loading was selfgenerated, forearm displacement was found to be $5^{\circ}$. In contrast, if the unloading was carried out by the experimenter, there was no anticipatory inhibition of the postural forearm flexors and when unloading did occur there was an upward rotation of the postural forearm by $16^{\circ}$ (Forget \& Lamarre, 1995; Hugon et al., 1982). If the time of the experimenter unloading was cued by a warning tone at a fixed interval before removal, there was still no anticipatory response in the forearm muscles. Therefore, self-generated movement was crucial for the anticipation to occur. Further studies demonstrate that not every active movement is able to result in an anticipatory adjustment. If the participant simply pressed a button with their thumb to remove the load, anticipation did not occur even after several hundred presentations of the situation, and the forearm displacement was large at $20^{\circ}$ (Duffose et al., 1985). Additionally, the anticipatory response did not transfer between limbs (Ioffe et al., 1996). If participants were first trained on an unloading task with a right active 
arm and a left postural arm and switched on testing to the right arm being the postural arm, no anticipatory response was found to occur. However, when the leg rather than the contralateral arm was used to postural limb in the unloading task, an anticipatory response occurred in the quadriceps, and postural disturbance was minimal at $6^{\circ}$ (Forget \& Lamarre, 1995).

\subsection{Grip force as a paradigm for forward models}

Grip-force modulation provides an ideal paradigm to study forward predictive model learning, due to the differing latency of response that occurs to alterations in load force that are self-generated compared to adjustments in load that are externally generated. This can then be extended to examine the formation of a forward model for a bi-manual manipulative task.

\subsubsection{Anticipatory grip force modulation}

When we hold an object in a precision grip between the thumb and forefinger, sufficient grip force (perpendicular to the surfaces) must be generated to prevent the object from slipping (Johansson \& Cole, 1992, 1994; Johansson, Riso, Hager, \& Backstrom, 1992; Johansson \& Westling, 1984). The level of grip force required depends on the load force (tangential to the surfaces) the object exerts on the fingers, that is its weight when at rest, and the frictional properties of the surface of the object. In general, participants avoid excessive grip forces; applying enough grip force to prevent the object slipping with grip force rarely exceeding the minimum grip force by more than 30\% safety margin (Johansson \& Cole, 1992). This strategy avoids both object breakage and unnecessary muscle fatigue.

When the object is moved by the participant's own action the load force on the fingers must change to accelerate the gripped object. Without a corresponding change in grip force the object would slip. In this self-generated situation the grip force tends to parallel load force with negligible delay.

Such anticipatory modulation is seen in discrete self-generated movements when pulling on an object in different directions and at different speeds (Johansson et al., 1992; Johansson \& Westling, 1984). Anticipation has also been shown to occur during continuous self-generated movements (Flanagan \& Wing, 1993, 1995). When we move an object by moving our arm, grip force modulates in phase with load force. Grip force increases as the load increases, and also falls as the load decreases. Despite the relatively rapid response of cutaneous afferents, such anticipatory control of grip force cannot be explained as a reaction to peripheral feedback (Flanagan \& Wing, 1995; Johansson \& Westling, 1984) due to unavoidable feedback delays (Forssberg et al., 1992; Johansson \& Westling, 1984). A system based solely on feedback control would be ineffective for manipulative actions with frequencies above about $1 \mathrm{~Hz}$, which would exclude some complex skills (Johansson \& Cole, 1994; Kunesch, Binkofski, \& Freund, 1989). This suggests that the skilled manipulation of objects requires the central nervous system (CNS) to use the motor command, in conjunction with internal models of both the body and the object, to anticipate 
the resulting load force and thereby adjust grip force appropriately (Blakemore, Goodbody, \& Wolpert, 1998; Flanagan \& Wing, 1997; Wing, Flanagan, \& Richardson, 1997).

\subsubsection{Reflexive grip force modulation}

The predictive response present in response to self-generated loads can be contrasted with the response when the load force is unexpectedly increased, for example by someone else tapping on the object. In this externally-generated situation a grip force response is triggered by the cutaneous receptors in the fingertips with a latency of around 60-100 ms after load force (Blakemore et al., 1998; Johansson et al., 1992; Johansson \& Westling, 1988). This reactive grip force response must depend on the feedback from cutaneous afferents as afferents from intrinsic and extrinsic hand muscles and the inter-phalangeal joints do not respond to alterations in load force fast enough (Macefield \& Johansson, 1996).

Therefore, the latency of the grip force response to loading can be used as an indicator of the degree of predictive learning.

\subsection{Inter-dependence in bi-manual grip force control: Symmetric loading}

To understand how our bi-manual internal models are learned it is first important to examine how grip force at each hand is co-ordinated when they simultaneously develop grip force to support concurrent bi-manual load force. This was recently addressed by (Bracewell, Wing, Soper, \& Clark, 2003) who examined both intra- and inter-manual co-ordination of grip force and load force during the bi-manual lifting and holding of a single object. They compared grip force coupling between hands on a voluntary task where a predictable load is lifted with a situation where unpredictable loadings were applied to a single object held bi-manually. The grip force rates generated by the two hands were correlated. In part, as would be expected, the correlation between the grip force rates across the two hands was due to the correlation between the load force rates at each hand. However, the grip force rates remained correlated when the effects of the correlation in load force rates were taken into account. Bracewell et al. (2003) found that the inter-manual correlation of grip force rates was in fact stronger than when the object held bi-manually was perturbed unpredictably compared with the correlation in the voluntary bi-manual lift. They suggest that the co-ordinative constraints within the specification of grip forces are at lower levels in the motor control hierarchy as they are more strongly related to the control of reflexive rather than predictive grip force (Bracewell et al., 2003).

This inter-dependence in the control of grip force between the two hands has been demonstrated in a number of other recent studies, for example Serrien and Wiesendanger (2001a). In their task participants were instructed to use both hands to lift two objects whose weights were changed. When the weight of an object was reduced, there was a decrease in grip force. The reduction in grip force was less when the contralateral hand was consecutively used to lift a heavy object. This study is consistent with the findings from a further recent study (Quaney, Rotella, Peterson, \& Cole, 
2003) questioning the scaling of predictive grip force response solely on object related properties. Their study demonstrates the inter-dependence of grip force control between the two hands. An 'unrelated' isolated pinch was found to influence the grip force applied on a subsequent lift trial of a hand held object to the same order of magnitude as a preceding, and 'related' lift trial of that object. There was no increase in the participant's load force that would be expected if the increase in grip force were explained through a generalized facilitation and the increase in applied grip force occurred if the 'unrelated' pinch or 'related' lift was made with the same or opposite hand (Quaney et al., 2003).

Further evidence for bi-manual inter-dependence in the control of grip force is shown in studies that use a bi-manual drawing opening task (Serrien \& Wiesendanger, 2001b). In this task the left hand pulled open a drawer whilst the right hand grasped an object. Grip/load force coordination was much higher in the bi-manual condition when the left hand was concurrently pulling the drawer, than in the unimanual condition, even though similar load forces were generated by the right hand in the two conditions. Additionally, comparisons between the grip force and load force ratios in two hands during uni-manual and bi-manual object lifting actions support the inter-dependence of grip force control between two hands. The weight of either hand's load, or the distance that either hand was required to move was compared to the associated grip force/load force ratios in the two hands in symmetrical and asymmetrical conditions. In uni-manual action force ratios were different under different load and distance conditions whereas in the bi-manual actions the load and distance of the other hand in the task influenced the force ratios.

\subsection{Bi-manual tasks to examine differing contributions in the development of prediction}

Two recent virtual object paradigms (Ohki, Edin, \& Johansson, 2002; Witney, Goodbody, \& Wolpert, 1999) are particularly useful in demonstrating the learning and adaptability of the forward predictive model that is thought to underlie anticipatory grip force modulation and also how the selection of a forward model may lead to the predictive learning of reflexive grip force responses (Ohki \& Watanabe, 2004). These studies enable an assessment of the relation between the neural control of reflexive and predictive grip force and how this inter-relationship may influence the adaptation of the motor system to manipulating novel objects.

Importantly, grip force modulation in bi-manual tasks can be used as a means of examining the basis for the formation of an internal model of a single physical object held between the hands. A number of factors are identifiable in increasing the learning of a prediction that a single object is being manipulated. First, the most common basis for predictive learning is an efference copy of the outgoing command. However, the development of prediction in this case can be due to an accurate knowledge of the timing of an event. The anticipation involved when a ball is being caught is an example of the learning of an internal model that does not rely on an efference copy of the motor command, but instead an internal model that is learned through a pre- 
cise knowledge of the timing of the occurrence of the event (Lacquaniti, 1996; Lacquaniti \& Maioli, 1989; Morton, Lang, \& Bastian, 2001). Within the paradigm of grip force modulation there are instances where these different means of developing predictions could be traded off. Accurate timing of a loading event, for instance the occurrence of synchronicity of loading between the two hands, may be sufficient for the formation of an internal model for a single object being manipulated. Accurate timing of a loading event could also be signaled by an external cue, for instance an auditory tone. This knowledge of the timing of a forthcoming loading event may be sufficient in the formation of an appropriate grip force prediction.

\section{Virtual object paradigm I: The use of a predictive model}

Forward predictive models capture the causal relation between action and outcome as signaled by efference copy. Using a bi-manual virtual object paradigm, it is possible to examine the learning of the relationship between action and outcome. Such a paradigm has allowed the separation of anticipatory grip force from the grip force due to reflexive processes, thereby allowing an estimate of the predictive response (Witney et al., 1999). To achieve this a bi-manual task has been used where each hand held a separate object each mounted on a torque motor.

A schematic diagram of the apparatus is shown in Fig. 1.

The forces generated by the motors were under computer control. This enabled 'virtual' objects to be created, where the forces experienced at each hand were varied on a trial by trial basis (Blakemore et al., 1998; Witney et al., 1999). On each trial, the

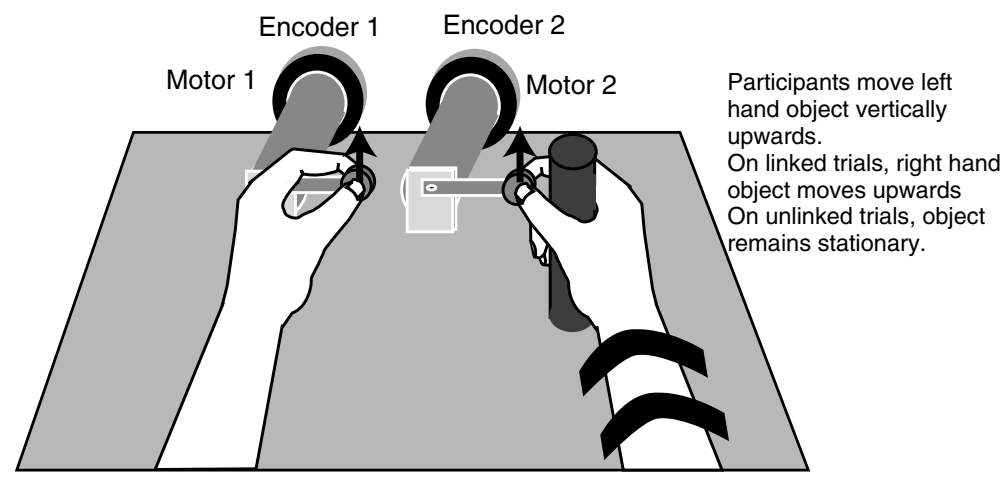

Fig. 1. The virtual object paradigm I. Schematic diagram of the apparatus used to create a virtual object. Each hand held an object that was attached to its own torque motor. The starting position of the participant in the trial is shown. During the trial, the participant was required to pull vertically up on the object held in the left hand and to maintain the position of the object held in the right hand. The torque motors were computer controlled so that the objects could be either 'linked', so that they acted as a single object, or 'unlinked', so that they acted as two independent objects. The participant's right forearm was anchored with velcro straps and for further stability, they grasped a vertically oriented aluminium rod with their three ulnar fingers. A horizontal wooden rod (not shown) was positioned over the right thumb and forefinger to minimize upward motion. 
participant had to generate a movement of the object held in the left hand. The motion of this left hand acted against a simulated stiff spring attached to the object's initial position. There were two possible consequences of this action of the left hand on the object held in the right hand. In "linked" trials the motion of the left hand caused an equal and opposite force to be generated on the object in the right hand independent of the right hand object's position. Therefore, during 'linked trials' the system behaved as though there were a real physical object held between the hands with each hand experiencing an equal and opposite force. In "Unlinked" trials, no force was generated on the right hand object. Therefore, on unlinked trials the two objects behaved independently, and so predictive grip force modulation in the right hand was not appropriate. On both linked and unlinked trials the load forces generated on the left hand object was the same. The grip force was examined in the right hand with the expectation that linked trials would result in predictive grip force modulation, and unlinked trials may suppress predictive grip force modulation. The unlinked trials were used to assess the development of the forward model. In these trials, any changes in grip force seen in the right hand reflects a purely predictive component as there was no load force generated on the fingers of this hand. Therefore, grip force modulation occurring in unlinked trials must have been as a consequence of a predictive model.

First, a series of experiments will be reviewed that have used this virtual object paradigm to examine various aspects of the predictive learning of grip force when loading is self-generated (Witney, Goodbody, \& Wolpert, 2000; Witney, Vetter, \& Wolpert, 2001). This will then be compared to a similar paradigm used to examine the predictive learning in reflexive grip force and the development of an internal model for a single object being manipulated between two hands (Ohki et al., 2002; Ohki \& Watanabe, 2004).

\subsection{Development and decay of grip force prediction}

Object properties are learnt through development, indicated by increasing ability to correctly parameterize grip force to the object being manipulated (Eliasson, Gordon, \& Forssberg, 1995; Forssberg, Eliasson, Kinoshita, Johansson, \& Westling, 1991; Forssberg, Eliasson, Kinoshita, Westling, \& Johansson, 1995; Forssberg et al., 1992). By the end of 2 years, children's grip and load forces begin to increase in parallel, with this coupling increasing to adult values by 8 years, through improvements can occur as late as 14 years (Eliasson et al., 1995; Forssberg et al., 1991; Forssberg et al., 1995; Forssberg et al., 1992). Several studies have examined how adult participants perform when the normal physical properties of an object, such as its weight, frictional surfaces, and surface orientation, are changed on each trial (Birznieks, Burstedt, Edin, \& Johansson, 1998; Edin, Westling, \& Johansson, 1992; Gordon, Westling, Cole, \& Johansson, 1993; Jenmalm \& Johansson, 1997; Johansson \& Westling, 1984). These show a rapid updating within one trial to the new situation. 
The causal relation between action and consequence represented by a forward model is not innate. In addition, through life this relationship will alter as the limbs grow or the objects encountered have different properties. Therefore, to examine adaptation of predictive learning in adults, Witney et al. (2000) examined the development of a predictive grip force response to the situation of a single object being manipulated bi-manually. By using the previously described 'virtual object' paradigm, the time course of development and decay of a predictive response that a single object was being manipulated between two hands could be examined. In this study, when participants made a short upward pulse with their left hand at the start of a trial, "linked" or "unlinked" trials were experienced. In a linked trial the objects behaved as a single stiff object held between the hands, with the motion of the left hand producing equal and opposite load forces on the two hands. In unlinked trials, the two objects behaved independently. Four different situations were compared to study the development and decay of a predictive grip force response. In all conditions, the grip force response during unlinked trials that followed a linked trial was examined.

After a single linked trial, anticipatory grip force developed, and grip force modulation continued to be present over the following unlinked trials. The peak grip on the first unlinked trial was $4.8 \mathrm{~N}$, and this decayed to $3.2 \mathrm{~N}$ by the sixth unlinked trial (Fig. 2(a)). A condition was included to examine the effect of varying the time between trials. This showed that the rate of decay depended on the number of trials
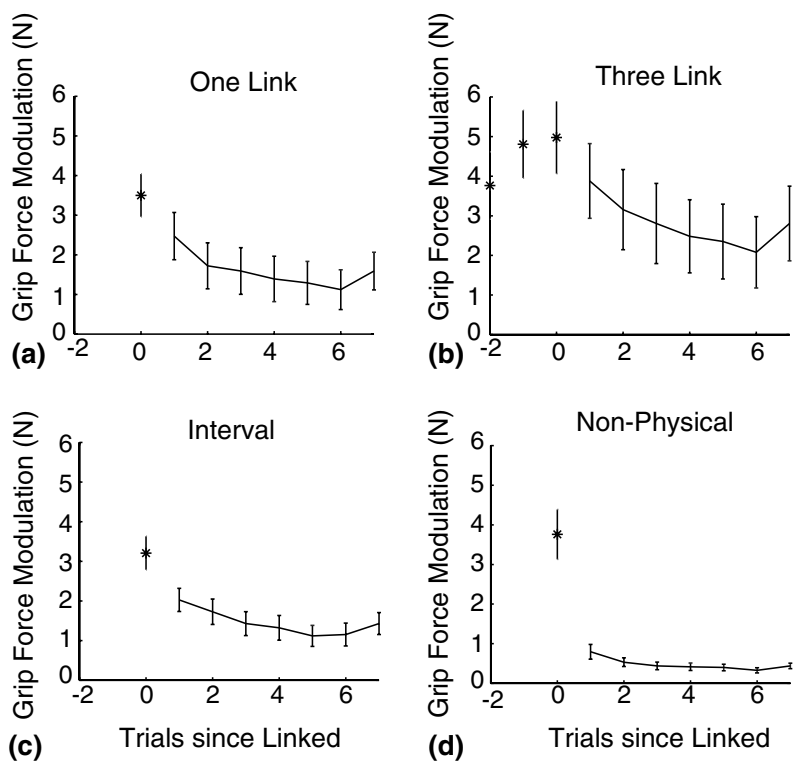

Fig. 2. The development and decay of predictive grip force modulation. Average of the participant's mean grip force modulation (a-d). Linked trials (star) and unlinked trials (solid) as a function of the number of trials since the last linked trial. (a) One link condition. (b) Three link condition. (c) Interval Condition. (d) Non-physical condition. Error bars show one standard error. Adapted from (Witney et al., 2000). 
since the last linked trial rather than time (Fig. 2(b)). Increasing the frequency of linked trials showed an increased level of subsequent grip force modulation, but did not alter the decay rate (Fig. 2(c)). When the torque motors simulated a linked object that did not have normal physical properties, prediction was reduced (Fig. $2(d)$ ). These results show that the use of predictive responses has a different time course for learning and decay, and the response depends on experience and the physical properties of the objects.

Examination of the grip force response on linked trials (single object) and unlinked trials (two separate objects) showed there were differences in both the magnitude and lag of these trial types. The grip force response on linked trials was found to be both delayed in timing and increased in magnitude compared to the grip force response on unlinked trials. These differences can be attributed to the feedback process, that is a comparison of the predicted (from the forward model) and actual sensory feedback that leads to the curtailing the feed-forward response in the absence of the predicted sensory feedback.

Predictive grip force manipulation was found to build up quickly, but decay slowly (Fig. 2). However, the association between action of one hand and the consequence of a load force on the other was found not to be sufficient to generate a predictive response. For a predictive response to develop it was necessary for the forces between the two hands to behave as a real physical object with equal forces to each hand Witney et al. (2000). This is comparable to findings in unloading tasks where self-generation alone is not the sufficient for anticipation to occur (Blakemore et al., 1998; Massion, 1992; Nowak \& Hermsdorfer, 2003). Further work could examine the similarities between prediction in the unloading task and in this task, including bi-manual transfer of prediction (Ioffe et al., 1996). The speed of decay of prediction is important in illustrating the flexibility associated with that aspect of the grip force response.

Further insight into predictive learning of a single object being manipulated between the hands, as opposed to two separate objects may come from insights provided by a recent study. The concept of a utility or loss function has now been applied to sensori-motor learning (Kording \& Wolpert, 2004b). Utility describes an individual's preference for a given circumstance, and has its origins in game theory (Von Neumann \& Morgenstern, 1944). When this concept is applied to sensorimotor control, a loss function during motor learning implies that the CNS has a measure of the relative success or cost of a movement that is made based on some function of the task. During motor learning, changes in performance are thought to occur through the optimisation of some function of the task; the loss function and this is how the CNS rates the relative success or cost of an executed movement (Kording \& Wolpert, 2004b).

Within grip force, there is the important goal of preventing the object from slipping from our grasp and dropping to the floor, and potentially breaking. Therefore, there may be an inherent preference for the predictive learning of a single object being held between the hands rather than two separate objects being manipulated due to the utility of maintaining the object's position. At the same time, it may be 
essential that our grip force does not rise too high as we may crush the object, or become fatigued. Under certain circumstances and task demands, for instance to hold a raspberry without causing damage to the fruit, it may be that is the careful handling of the object that is the utility function and this may influence the level of grip force applied. It could also be argued that other factors, including externally imposed ones, could increase or decrease the preference for the manipulated objects to be regarded as a single object or as two separate objects.

An important question that remains with this formulation is what is the depth of previous experience that is accounted for in the generation of a future prediction? It might be in this virtual object paradigm that a single occurrence of slippage, if this is the utility function that the participant is optimising, may lead to a much increased weighting towards predictive learning of a single object than if the object is held successfully without any slippage during 'linked' trials.

\subsection{The effect of previous experience on predictive control}

Statistical approaches have become important in understanding how predictions develop to altering environments (Davidson \& Wolpert, 2003). These ideas emphasize how prior predictions are integrated with current sensory information for optimal motor output, with Bayesian integration being used to model experimental results (Kording \& Wolpert, 2004a). How do prior predictions influence current motor output in the predictive learning of a single object being manipulated between the hands?

Previous experience of manipulation has been shown to influence the current grip force response, with predictions of objects properties based on those experienced in the preceding trial as long as there were not alterations in the visual features of the manipulated object (Gordon et al., 1993; Johansson \& Cole, 1992), with this effect occurring even if the weight of the experimental object was varied unpredictably (Forssberg et al., 1992). This effect of previous experience was further examined through the analysis of sequential effects (Witney et al., 2001) in the same virtual object paradigm. Participants held the two objects bi-manually and were requested to make a movement vertically upwards with their left hand. On linked trials a loading was applied to the contralateral object and on unlinked trials no loading was applied. A pseudo-random series of linked and unlinked trials were presented to participants. Different proportions of linked and unlinked trials were presented to participants (30 $\%, 50 \%$ and $70 \%$ ). The effect of manipulative history on the current prediction was assessed by examining grip force prediction on the unlinked trials, where no loading occurred to the object, and therefore any grip force modulation must be in anticipation of the association between the action of one hand and consequence of this action on the other. The predictive grip force modulation showed a systematic influence of the experience of linked or unlinked trials on the prediction that a single object was being manipulated. Such effects are thought to be characteristic of a process that is dependent on an underlying automatic process and has been found in anticipatory eye movements (Kowler, Martins, \& Pavel, 1984). 
Grip force prediction regarding whether the object was linked and behaving like a single object held between each hand or unlinked, and behaving like two separate objects, was dependent on the weighted average of the object's properties as experienced over at least the last three trials. In this paradigm, the influence of purely cutaneous information was examined, and demonstrates how there is a short-term memory for previous experience that explains the current prediction. However, if this uncertain manipulative experience were combined with sensory information from other sources, for instance vision, very different results could be expected as visual information may dominate under these circumstances. Further work is needed to examine this question.

\section{Virtual object paradigm II: The development of prediction to bi-manual external loadings}

Grip force adjustments during object manipulation are also driven through feedback control, and it is interesting to examine whether feedback from cutaneous afferents alone can lead to the development of predictive grip force modulations appropriate for a single object being manipulated between two hands. When external loadings occur on a grasped object, there is a reflexive modulation of grip force to maintain object stability. When we are holding a single object between two hands, reflexive grip force modulation will occur in each hand when an external perturbation is applied to the object. It is interesting therefore, to compare the reflexive modulation of grip force in this context to a situation where external loadings are applied to two separate objects, one held in each hand, as this can be used to examine how the occurrence of synchronous cutaneous inputs can lead to an internal model for a single object being manipulated bi-manually being evoked.

When we hold an object in both hands the sensory information from a digit induces an automatic response not only in the ipsilateral hand but also in the contralateral hand (Ohki \& Johansson, 1999). The development of prediction to external loadings has been examined using a bi-manual virtual object paradigm (Ohki et al., 2002; Ohki \& Johansson, 1999; Ohki \& Johansson, 2000; Ohki \& Watanabe, 2004). Some of these studies have paralleled the experimental design of (Witney et al., 2000) and analysis of (Witney et al., 2001) thus enabling interesting comparisons to be made with the development of predictions to self-generated loadings and also the effect of previous experience. These recent studies show that anticipation developed to this situation of external loadings being applied synchronously to each hand. This adaptation cannot rely on prediction developed by the use of an efference copy of the motor command. This therefore raises questions regarding the development of predictive learning. When an internal model is formed of a single object being manipulated bi-manually. First, what is the extent of the involvement of efference copy? Second, what is the relative importance of this information with the situation of exactly synchronous loadings occurring to each hand?

The bi-manual task that was used in some of the studies, for instance (Ohki et al., 2002) was a virtual object paradigm where there were two grasp plates that were each 


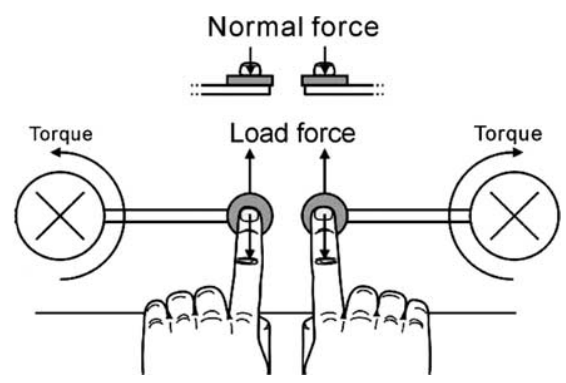

Fig. 3. The virtual object paradigm II. Schematic diagram of the apparatus used to create a loading situation that simulates the loading experienced when a single object is held between two hands and pulled on by a second person (Ohki et al., 2002). The virtual object paradigm comprised of two flat circular grasp plates that were each connected to separate servo regulated torque motors via a beam. The normal (grip) and tangential load force on both grasp surfaces were measured.

connected to separate servo regulated torque motors via a stiff rod (Fig. 3). Each grasp surface was under computer control, and this enabled the forces at each finger to be altered on a trial-by-trial basis. Therefore, the presentation of a series of trials where the grasp surfaces at each hand are simultaneously loaded was possible. Concurrent loading on the two grasp plates provides a simulation of the loading situation where a single object held between both hands is pulled by a second person.

Ohki et al. (2002) observed that pre-load grip force and grip force response became adapted to a loading condition when participants experienced the condition during sequentially presented trials. However, the grip force response that was present immediately after an unexpected change of loading conditions was influenced by the previous loading condition. Several loading trials of the same type were required for the participant to display complete adaptation to the new condition, which is a similar finding to (Witney et al., 2000).

Ohki et al. (2002) argue that if the internal model appropriate for the context of a single object held between two hands can modulate the neural centre that is involved in reflexive grip force adjustments, the adaptation of the reflexive response would be controlled in a similar way to the adaptation of prediction in voluntary movements. Therefore, similarities might be expected in adaptation of the reflexive grip force response with the findings of (Witney et al., 2000) examining adaptation of prediction of voluntary movements. To examine this question Ohki and Watanabe (2004) presented participants with a similar series of trials as in Witney et al. (2000). Linked trials in these experiments consisted of simultaneous external loadings to left and right fingers, and were followed by a series of unlinked trials where loading occurred to one side only. Additionally there were different durations of waiting phase between trials, giving a long and short interval series, in order for comparisons to be made with the influence of an increased interval in self-generated loadings (Witney et al., 2000).

There were clear effects in the amplitude of the response over sequential unlinked trials. When load condition changed from concurrent loading to isolated loading, the 
response of the unloaded finger was high in the first trial and significantly reduced over subsequent unlinked trials, until there was little response after the experience of seven unlinked trials. This adaptive decay occurred regardless of the number of previously experienced linked trials that preceded the linked trials and regardless of whether the interval between the trials was long or short. Earlier work suggests that grip force adaptation is event driven (Johansson \& Cole, 1992) and therefore, the time between subsequent events should not be as important an influencing factor in the adaptation of grip force responses. Similarities were found in the influence of short and long interval series between loading trials in the Ohki and Watanabe (2004) and Witney et al. (2000) paradigms (Fig. 4). Adaptation was found in both instances to depend on feedback events that occurred, but not the duration of time after an event.

Additionally, the effect of previous experience of external loadings was examined. Sensory feedback of a loading or unloading was found to influence future grip force

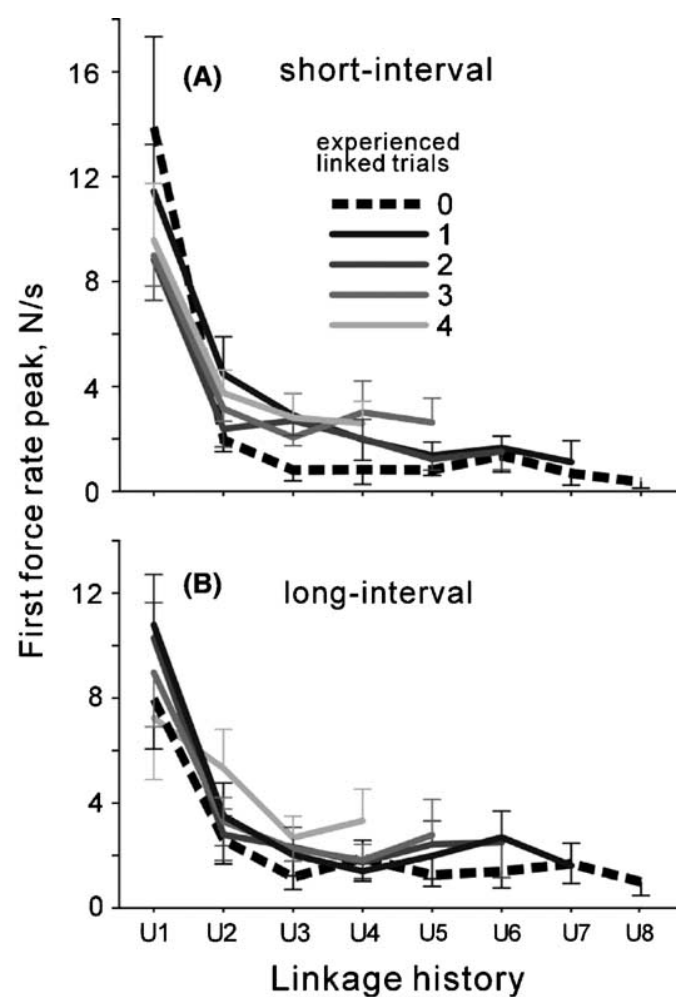

Fig. 4. The effect of linkage on grip force. Effects of preceding linked trials during sequential unlinked trials. First force rate peaks in sequential unlinked trials are shown for non-loaded right fingers and obtained in link-predictive test series. Values are presented separately with lines in different styles depending on the number of experienced linked trials after the beep, which preceded unlinked trials, from Ohki and Watanabe (2004). 
response in a way in which prediction error determines modification of the response in both the Ohki and Watanabe (2004) and Witney et al. (2000) paradigms. Therefore, Ohki and Watanabe (2004) demonstrate that the selection of an internal model for a single object being held bi-manually and pulled by a second person can modulate the neural centre processing sensory driven reflexive responses due to the presence of this higher order representation.

Further work is needed to examine the behaviourally evidenced asymmetries in learning and the underlying neural control to gain a complete understanding of the neural substrates underlying the control of bi-manual actions. When we hold an object in both hands the sensory information from the cutaneous receptors on a digit induces an automatic response not only in the ipsilateral hand but also in the contralateral hand (Ohki \& Johansson, 1999). This suggests that cutaneous inputs can activate ipsilateral motor cortex as well as contralateral motor cortex. The importance of ipsilateral corticospinal control may have a greater role than previously thought (Haaland \& Harrington, 1996). This role has been shown both in patient studies and also anatomical work. Interestingly, a recent Transcranial Magnetic Stimulation (TMS) study was performed using a virtual object paradigm. It was found that activation in the ipsilateral M1 was stronger after linked trials compared with unlinked trials where the situation of a single object being manipulated between two hands and pulled on by a second person is simulated (Shibuya \& Ohki, 2004).

\section{Adaptation without feedback experience}

Ohki and Watanabe (2004) showed that there are top-down influences on the adaptation of grip force responses to changing loading conditions. When an auditory cue was provided to warn the participant that there would shortly be a sudden switch in loading condition the participant could modify the grip force response. However, these effects on grip force adaptation were found to be weak compared with the influence of feedback from cutaneous afferents in adapting the grip force response on a subsequent trial. With self-generated movement, visual information is a further sensory modality that is known to affect the anticipatory parameterization of fingertip forces. This is powerfully demonstrated in the size-weight illusion when participants encounter misleading visual size cues about the weight of an object they continue to influence predictive force control for a number of trials despite somatosensory information before grip force does become appropriately scaled (Flanagan \& Beltzner, 2000). Therefore, the influence of visual cues is much stronger than the influence of auditory cues in the scaling of grip force predictions. This difference between sensory modalities could be that visual size cues are frequently used in daily life to gauge the weight of an object whereas this is generally not the case for auditory cues. 


\section{Discussion}

The learning of bi-manual co-ordination is crucial in our performance of many everyday tasks, and the studies reviewed by this paper highlight the complexity underlying its development. Here the internal model perspective to bi-manual coordination is presented. Many existing studies of internal model learning have concentrated on the learning of new environments uni-manually. However, due the natural asymmetry in the control of our movements, with the corresponding neural asymmetries, the formation of an internal model for a bi-manual task must be a highly complex process. The learning of predictive grip force modulation is an important means of examining the properties of forward predictive models. In this paper a series of experiments was reviewed (Ohki et al., 2002; Witney et al., 2000; Witney et al., 2001) that examined the adaptation of anticipatory and reflexive grip force responses using virtual object paradigms. These studies demonstrate the usefulness of the grip force paradigm in examining prediction of object properties and more complex co-ordinative tasks. In these paradigms two situations were simulated, the forces on each object were controlled so that the two objects could be either 'linked' so that they acted as a single object, or 'unlinked', so that they acted as two independent objects. The loadings on the object were either self-generated, simulating the situation of a single physical object being manipulated between the two hands, or externally-generated, simulating the situation of a single physical object, held between the two hands being pulled on by a second person. A number of features of predictive grip force modulation learning were determined, including the time course of the development and decay of prediction. These characteristics of predictive learning may be explicable through a number of recently proposed ideas. First, that the current prediction is the result of the integration between current experience and prior predictions (Kording \& Wolpert, 2004a). Second, that loss functions drive motor learning (Kording \& Wolpert, 2004b). With the formation of an object representation these ideas provide explanations for both the shortterm weighted averaging process that occurs when presented with uncertain environment (Witney et al., 2001) and also the asymmetries that occur in the development of a prediction that a single object is being manipulated (Witney et al., 2000).

These studies demonstrate how synchronicity of loading is a key factor in the development of knowledge of an object (Ohki et al., 2002) as well as external cues, for instance auditory signals (Ohki \& Watanabe, 2004), in addition to efference copy of the motor command (Witney et al., 2000). When we handle objects, high order predictions about an object's dynamics are used to modify the response made to cutaneous feedback in a way most appropriate to the manipulative context. There were many similarities in the adaptation that occurs in grip force during external loading as to self-generated loading when a context is evoked. The recent proposal of a hierarchical multi-layered controller (Wolpert et al., 2003) provides a useful theoretical framework to further examine the predictive learning of a bi-manual coordinative task However, the precise influences of our demonstrated asymmetries 
in neural control on predictive learning in bi-manual tasks, and the differences in generalization properties dependent on hand dominance, leaves many questions still un-answered about how the predictive learning of object manipulation overcomes these inherent complexities.

\section{Acknowledgments}

AGW is a Wellcome Trust Advanced Training Fellow. Support has also been provided by the MRC, EU and ARC. Experimental work was conducted while an MRC graduate student of Prof. Daniel Wolpert, Sobell Dept of Motor Neuroscience, Institute of Neurology, London. Thanks also to Prof. Alan Wing and SyMoN Group, University of Birmingham. Thanks to Prof. Joachim Hermsdoerfer for inviting participation in the interesting EWOMS workshop "Anticipatory mechanisms in grip force control". Thanks to the two anonymous referees of this manuscript for their particularly helpful and constructive comments. Thanks also to Dr. Willem Verwey for helpful editorial comments.

\section{References}

Birznieks, I., Burstedt, M. K. O., Edin, B. B., \& Johansson, R. S. (1998). Mechanisms for force adjustments to unpredictable frictional changes at individual digits during two-fingered manipulation. Journal of Neurophysiology, 80, 1989-2002.

Blakemore, S. J., Goodbody, S. J., \& Wolpert, D. M. (1998). Predicting the consequences of our own actions: The role of sensorimotor context estimation. Journal of Neuroscience, 18, 7511-7518.

Bracewell, R. M., Wing, A. M., Soper, H. M., \& Clark, K. G. (2003). Predictive and reactive coordination of grip and load forces in bimanual lifting in man. European Journal of Neuroscience, 18, 2396-2402.

Carson, R. G., Chua, R., Elliott, D., \& Goodman, D. (1990). The contribution of vision to asymmetries in manual aiming. Neuropsychologia, 28, 1215-1220.

Carson, R. G., Goodman, D., Chau, R., \& Elliott, D. (1993). Asymmetries in regulation of visually guided aiming. Journal of Motor Behavior, 25, 21-32.

Criscimagna-Hemminger, S. E., Donchin, O., Gazzaniga, M., \& Shadmehr, R. (2003). Learned dynamics of reaching movements generalize from dominant to nondominant arm. Journal of Neurophysiology, $89,168-176$.

Davidson, P. R., \& Wolpert, D. M. (2003). Motor learning and prediction in a variable environment. Current Opinion in Neurobiology, 13, 232-237.

DiZio, P., \& Lackner, J. R. (1995). Motor adaptation to Coriolis force perturbation of reaching movements: Endpoint but not trajectory adaptation transfers to he nonexposed arm. Journal of Neurophysiology, 74, 1787-1792.

Duffose, M., Hugon, M., \& Massion, J. (1985). Postural forearm changes induced by predictable in time or voluntary triggered unloading in man. Experimental Brain Research, 60, 330-334.

Edin, B. B., Westling, G., \& Johansson, R. S. (1992). Independent control of human finger-tip forces at individual digits during orecision lifting. Journal of Physiology, London, 450, 547-564.

Eliasson, A. C., Gordon, A. M., \& Forssberg, H. (1995). Tactile control of isometric fingertip forces during grasping in children with cerebral palsy. Developmental Medicine and Child Neurology, 37, $72-84$.

Flanagan, J. R., \& Beltzner, M. A. (2000). Independence of perceptual and sensorimotor predictions in the size-weight illusion. Nature Neuroscience, 3, 737-741. 
Flanagan, J. R., \& Wing, A. M. (1993). Modulation of grip force with load force during point-to-point arm movements. Experimental Brain Research, 95, 131-143.

Flanagan, J. R., \& Wing, A. M. (1995). The stability of precision grip forces during cyclic arm movements with a hand-held load. Experimental Brain Research, 105, 455-464.

Flanagan, J. R., \& Wing, A. M. (1997). The role of internal models in motion planning and control: Evidence from grip force adjustments during movements of hand-held loads. Journal of Neuroscience, 17, 1519-1528.

Forget, R, \& Lamarre, Y. (1995). Postural adjustments associated with different unloadings of the forearm-effects of proprioceptive and cutaneous afferent deprivation. Canadian Journal of Physiology and Pharmacology, 73, 285-294.

Forssberg, H., Eliasson, A. C., Kinoshita, H., Johansson, R. S., \& Westling, G. (1991). Development of human precision grip. 1. Basic coordination of force. Experimental Brain Research, 85, 451-457.

Forssberg, H., Eliasson, A. C., Kinoshita, H., Westling, G., \& Johansson, R. S. (1995). Development of human precision grip. 4. Tactile adaptation of isometric finger forces to the frictional condition. Experimental Brain Research, 104, 323-330.

Forssberg, H., Kinoshita, H., Eliasson, A. C., Johansson, R. S., Westling, G., \& Gordon, A. M. (1992). Development of human precision grip. 2. Anticipatory control of isometric forces targeted for objects weight. Experimental Brain Research, 90, 393-398.

Goodbody, S. J., \& Wolpert, D. M. (1998). Temporal and amplitude generalization in motor learning. Journal of Neurophysiology, 79, 1825-1838.

Gordon, A. M., Westling, G., Cole, K. J., \& Johansson, R. S. (1993). Memory representations underlying motor commands used during manipulation of common and novel objects. Journal of Neurophysiology, 69, 1789-1796.

Haaland, K. Y., \& Harrington, D. L. (1996). Hemispheric asymmetry of movement. Current Opinion in Neurobiology, 6, 796-800.

Haruno, M., Wolpert, D. M., \& Kawato, M. (2001). MOSAIC model for sensorimotor learning and control. Neural Computation, 13, 2201-2220.

Hugon, M., Massion, J., \& Wiesendanger, M. (1982). Anticipatory postural changes induced by active unloading and comparison with passive unloading in man. Pflügers Archives, 393, 292-296.

Ioffe, M., Massion, J., Gantchev, N., Duffose, M., \& Kulikov, M. A. (1996). Coordination between posture and movement in a bimanual load lifting task-is there transfer. Experimental Brain Research, $109,450-456$.

Jenmalm, P., \& Johansson, R. S. (1997). Visual and somatosensory information about object shape control manipulative fingertip forces. Journal of Neuroscience, 17, 4486-4499.

Johansson, R. S., \& Cole, K. J. (1992). Sensory-motor coordination between grasping and manipulative actions. Current Opinion in Neurobiology, 2, 815-823.

Johansson, R. S., \& Cole, K. J. (1994). Grasp stability during manipulative actions. Canadian Journal of Physiology and Pharmacology, 72, 511-524.

Johansson, R. S., Riso, R, Hager, C., \& Backstrom, L. (1992). Somatosensory control of precision grip during unpredictable pulling loads. 1. Changes in load force amplitude. Experimental Brain Research, 89, 181-191.

Johansson, R. S., \& Westling, G. (1984). Roles of glabrous skin receptors and sensorimotor memory in automatic-control of precision grip when lifting rougher or more slippery objects. Experimental Brain Research, 56, 550-564.

Johansson, R. S., \& Westling, G. (1988). Programmed and triggered actions to rapid load changes during precision grip. Experimental Brain Research, 71, 72-86.

Jordan, M. I. (1996). Computational aspects of motor control and motor learning. In H. Heuer \& S. Keele (Eds.), Handbook of perception and action: Motor skills (pp. 71-120). New York: Academic Press.

Kawato, M., Furawaka, K., \& Suzuki, R. (1987). A hierarchical neural network model for the control and learning of voluntary movements. Biological Cybernetics, 56, 1-17.

Kelso, J. A. S., Southard, D. L., \& Goodman, D. (1979). On the coordination of two-handed movements. Journal of Experimental Psychology: Human Perception and Performance, 2, 229-238. 
Kording, K. P., \& Wolpert, D. M. (2004a). Bayesian integration in sensorimotor learning. Nature, 427, 245-247.

Kording, K. P., \& Wolpert, D. M. (2004b). The loss function of sensorimotor learning. Proceedings of the National Academy of Sciences, 101, 9839-9842.

Kowler, E., Martins, A. J., \& Pavel, M. (1984). The effect of expectations on slow oculomotor control. IV: Anticipatory smooth eye movements depend on prior target motions. Vision Research, 24, 197-210.

Kunesch, E., Binkofski, F., \& Freund, H.-J. (1989). Invariant temporal characteristics of manipulative hand movements. Experimental Brain Research, 78, 539-546.

Lacquaniti, F. (1996). Neural control of limb mechanics for visuomanual coordination. In A. M. Wing, P. Haggard, \& J. R. Flanagan (Eds.), The neurophysiology and psychology of hand movements (pp. 213-239). New York: Academic Press.

Lacquaniti, F., \& Maioli, C. (1989). The role of preparation in tuning anticipatory and reflex responses during catching. Journal of Neuroscience, 9, 134-148.

Lacquaniti, F., Soechting, J. F., \& Terzuolo, C. A. (1982). Some factors pertinent to the organization and control of arm movements. Brain Research, 252, 394-397.

Lum, P. S., Reinkensmeyer, D. J., Lehman, S. L., Li, P. Y., \& Stark, L. W. (1992). Feedforward stabilization in a bimanual unloading task. Experimental Brain Research, 89, 172-180.

Macefield, V. G., \& Johansson, R. S. (1996). Control of grip force during restraint of an object held between finger and thumb: Responses of muscle and joint afferents from the digits. Experimental Brain Research, 108, 172-184.

Massion, J. (1992). Movement, posture and equilibrium: Interaction and coordination. Current Opinion in Neurobiology, 38, 35-56.

Morton, S. M., Lang, C. E., \& Bastian, A. J. (2001). Inter- and intra-limb generalization of adaptation during catching. Experimental Brain Research, 141, 438-445.

Nowak, D. A., \& Hermsdorfer, J. (2003). Sensorimotor memory and grip force control: Does grip force anticipate a self-produced weight change when drinking with a straw from a cup?. European Journal of Neuroscience, 18, 2883-2892.

Ohki, Y., Edin, B. B., \& Johansson, R. S. (2002). Predictions specify reactive control of individual digits in manipulation. Journal of Neuroscience, 22, 600-610.

Ohki, Y., \& Johansson, R. S. (1999). Sensorimotor interactions between pairs of fingers in bimanual and unimanual manipulative tasks. Experimental Brain Research, 127, 43-53.

Ohki, Y., \& Johansson, R. S. (2000). Reactive finger responses are influenced by grasp motor set prepared for objects' behaviour. European Journal of Neuroscience, 12, 197.

Ohki, Y., \& Watanabe, K. (2004). Dependence of reactive responses in human bimanual finger movements on sensory feedback and auditory cues. Journal of Neurophysiology, 91, 1260-1270.

Oldfield, R. C. (1971). The assessment and analysis of handedness: The Edinburgh Inventory. Neuropsychologia, 9, 97-113.

Ostry, D. J., \& Feldman, A. G. (2003). A critical evaluation of the force control hypothesis in motor control. Experimental Brain Research, 153, 275-288.

Paulignan, Y., Duffose, M., Hugon, M., \& Massion, J. (1989). Acquisition of coordination between posture and movement in a bimanual task. Experimental Brain Research, 77, 337-348.

Quaney, B. M., Rotella, D. L., Peterson, C., \& Cole, K. J. (2003). Sensorimotor memory for fingertip forces: Evidence for a task independent motor memory. Journal of Neuroscience, 23, 1981-1986.

Sainburg, R. L., \& Kalakanis, D. (2000). Differences in control of limb dynamics during dominant and nondominant arm reaching. Journal of Neurophysiology, 83, 2661-2675.

Sainburg, R. L., \& Wang, J. (2002). Interlimb transfer of visuomotor rotations: Independence of direction and final position information. Experimental Brain Research, 145, 437-447.

Salimi, I., Hollender, I., Frazier, W., \& Gordon, A. M. (2000). Specificity of internal representations underlying grasping. Journal of Neurophysiology, 84, 2390-2397.

Serrien, D. J., \& Wiesendanger, M. (2001a). Bimanual organization of manipulative forces: Evidence from erroneous feedforward programming of precision grip. European Journal of Neuroscience, 13, $1825-1832$. 
Serrien, D. J., \& Wiesendanger, M. (2001b). Dissociation of grip/load-force coupling during a bimanual manipulative assignment. Experimental Brain Research, 136, 417-420.

Shadmehr, R., \& Brushers-Krug, T. (1997). Functional stages in the formation of long-term motor memory. Journal of Neuroscience, 17, 409-419.

Shadmehr, R., \& Mussa-Ivaldi, F. A. (1994). Adaptive representation of dynamics during learning of a motor task. Journal of Neuroscience, 14, 3208-3234.

Shibuya, S., \& Ohki, Y. (2004). Cutaneous inputs can activate the ipsilateral primary motor cortex during bi-manual sensory-driven movements in humans. Journal of Neurophysiology.

Sperry, R. W. (1950). Neural basis of the spontaneous optokinetic response produced by visual inversion. Journal of Comparative Physiology and Psychology, 43, 482-489.

Swinnen, S. P., Walter, C. B., Lee, T. D., \& Serrien, D. J. (1993). Acquiring bimanual skills: Contrasting forms of information feedback for interlimb decoupling. Journal of Experimental Psychology: Learning, Memory \& Cognition, 19, 1328-1344.

Swinnen, S. P., \& Wenderoth, N. (2004). Two hands, one brain: Cognitive neuroscience of bimanual skill. Trends in Cognitive Sciences, 8, 18-25.

Von Holst, E. (1954). Relations between the central nervous systems and the peripheral organ. British Journal of Animal Behaviour, 2, 89-94.

Von Neumann, J., \& Morgenstern, O. (1944). Theory of games and economic behavior. Princeton, NJ: Princeton University Press.

Wang, J., \& Sainburg, R. L. (2003). Mechanisms underlying interlimb transfer of visuomotor rotations. Experimental Brain Research, 149, 520-526.

Wang, J., \& Sainburg, R. L. (2004). Interlimb transfer of novel inertial dynamics is asymmetrical. Journal of Neurophysiology, 92, 349-360.

Wing, A. M., Flanagan, J. R., \& Richardson, J. (1997). Anticipatory postural adjustments in stance and grip. Experimental Brain Research, 116, 122-130.

Witney, A. G., Goodbody, S. J., \& Wolpert, D. M. (1999). Predictive motor learning of temporal delays. Journal of Neurophysiology, 82, 2039-2048.

Witney, A. G., Goodbody, S. J., \& Wolpert, D. M. (2000). Learning and decay of prediction in object manipulation. Journal of Neurophysiology, 84, 334-343.

Witney, A. G., Vetter, P., \& Wolpert, D. M. (2001). The influence of previous experience on predictive motor control. Neuroreport, 12, 649-653.

Wolpert, D. M. (1997). Computational approaches to motor control. Trends in Cognitive Sciences, 1, 209-216.

Wolpert, D. M., \& Flanagan, J. R. (2001). Motor prediction. Current Biology, 11, R729-R732.

Wolpert, D. M., \& Ghahramani, Z. (2000). Computational principles of movement neuroscience. Nature Neuroscience, 3, 1212-1217.

Wolpert, D. M., \& Kawato, M. (1998). Multiple paired forward and inverse models for motor control. Neural Networks, 11, 1317-1329.

Wolpert, D. M., Doya, K., \& Kawato, M. (2003). A unifying computational framework for motor control and social interaction. Philosophical Transactions of the Royal Society of London Series B-Biological Sciences, 358, 593-602.

Wolpert, D. M., Ghahramani, Z., \& Flanagan, J. R. (2001). Perspectives and problems in motor learning. Trends in Cognitive Sciences, 5, 487-494. 